\title{
Uma proposta experimental de baixo custo para o estudo de oscilações amortecidas em regime subcrítico com auxílio de smartphone
}

\section{A low cost experimental proposal for the study of damped oscillations in subcritical regime using smartphone}

\begin{abstract}
M. A. V. Macedo Junior ${ }^{* 1}$, V. M. FragA ${ }^{1}$, G. Rousseau ${ }^{2}$
${ }^{1}$ Grupo de Pesquisa em Física e Ensino de Física, Instituto Federal de Educação, Ciência e Tecnologia do Rio de Janeiro, Campus Duque de Caxias, Avenida República do Paraguai, 120; CEP 25.050-100, Duque de Caxias, RJ, Brasil.

${ }^{2}$ Universidade Federal Fluminense, Rua Mário Santos Braga, s/n; CEP 24.020-140, Niterói, RJ, Brasil.
\end{abstract}

\begin{abstract}
Resumo
O smartphone é um dispositivo tecnológico capaz de realizar diferentes tarefas que vão desde a simples comunicação entre duas pessoas até a localização através do GPS, recursos biométricos de identificação por retina e digitais, registro de frequência cardíaca, dentre outros. O desenvolvimento e a constante evolução das tecnologias presentes nos smartphones proporcionaram o barateamento e a diminuição dos sensores nele presentes, permitindo assim, a popularização dessas ferramentas de medição. Esses sensores, com o auxílio de aplicativos adequados, podem ser utilizados para fins didáticos visto que, possibilitam a aquisição automática de dados de diferentes grandezas físicas. No presente trabalho apresentamos uma proposta de atividade experimental com materiais de baixo custo e uso cotidiano, como uma mola, uma régua e um smartphone, para o estudo de oscilações amortecidas em regime subcrítico. O objetivo é mostrar que apesar da simplicidade dos materiais é possivel se obter resultados satisfatórios a partir de um experimento didático, mesmo na ausência de sofisticados equipamentos de laboratório. Com as medidas da aceleração em função do tempo nos três eixos espaciais, obtidas pelo aplicativo Sensor Kinetics, foi possível calcular os valores da constante de amortecimento e da constante elástica da mola, além da construção dos gráficos das envoltórias superiores e inferiores da posição, velocidade e aceleração que modelam o movimento oscilatório.
\end{abstract}

Palavras-chave: Oscilações amortecidas. Regime subcrítico. Acelerômetro. Smartphone. Ensino de física.

*marcelo.junior@ifrj.edu.br 


\begin{abstract}
The smartphone is a technological device capable of performing different tasks ranging from simple communication between two people to the location through GPS, biometric features of retina and digital identification, heart rate recording, among others. The development and constant evolution of the technologies present in the smartphones provided the cheapening and the reduction of the sensors present in it, thus allowing the popularization of these measurement tools. These sensors, with the aid of suitable applications, can be used for didactic purposes since they allow the automatic acquisition of data of different physical quantities. In the present work we present a proposal of experimental activity with materials of low cost and daily use, such as a spring, a ruler and a smartphone, for the study of subcritical damped oscillations. The objective is to show that despite the simplicity of the materials it is possible to obtain satisfactory results from a didactic experiment, even in the absence of sophisticated laboratory equipment. With the acceleration measures as a function of time in the three spatial axes, obtained by the application Sensor Kinetics, it was possible to calculate the values of the damping constant and the elastic spring constant, as well as the construction of graphs of the upper and lower asymptotes of the position, speed and acceleration that model the oscillatory movement.
\end{abstract}

Keywords: Damped oscillations. Subcritical regime. Accelerometer. Smartphone. Physics teaching.

\title{
I. INTRODUÇÃO
}

O smartphone é um dispositivo tecnológico capaz de realizar diferentes tarefas que vão além das relacionadas à comunicação entre duas pessoas. Localização através do GPS, recursos biométricos de identificação por retina e digitais, registro de frequência cardíaca, são apenas algumas das funções presentes nos aparelhos smartphones atuais. Executar essas funções é possível visto que, apesar de seu tamanho e beleza exterior, os smartphones possuem um conjunto de poderosos sensores capazes de realizar sofisticadas medições.

Dentre os principais sensores existentes no smartphone o acelerômetro se destaca por estar presente até nas versões mais baratas dos aparelhos. De forma simples, podemos dizer que o acelerômetro é um sensor capaz de medir a aceleração provocada por forças que atuam no sensor (MONTEIRO et al., 2015). Essas forças podem ser estáticas, como a força da gravidade, ou dinâmicas causadas pela movimentação ou vibração do acelerômetro. Aparelhos de smartphone que possuem um acelerômetro para cada eixo, triaxiais, são capazes de determinar a orientação que o aparelho se movimenta no espaço, visto que cada eixo é fixo em relação ao smartphone (SILVA; SILVA, 2010). Além da orientação, os acelerômetros permitem ainda que seja mensurada a magnitude da aceleração ao longo de um eixo.

O desenvolvimento e constante evolução das tecnologias presente nos smartphones proporcionaram o barateamento e a diminuição dos sensores, permitindo assim a popularização dessas ferramentas de medição. Esses sensores, com o auxílio de aplicativos adequados, podem ser utilizados para fins didáticos visto que, permitem ao professor reproduzir experimentos científicos com alta precisão mesmo em instituições que não possuem laboratórios 
didáticos adequados para a realização de atividades experimentais. Na última década, diversos estudos foram realizados testando e apresentando os sensores do smartphone como ferramenta didática nas principais áreas abordadas no ensino de física, a saber: acústica, oscilações, ondas, ótica, mecânica, eletromagnetismo dentre outras (HAWLEY; MCCLAIN, 2018; IGOE et al., 2018; MONTEIRO et al., 2017; PEREIRA et al., 2017; SALINAS et al., 2018; VOGT; KUHN, 2012a).

No que se refere ao estudo de oscilações alguns temas relacionados já foram abordados na literatura, como é o caso do pêndulo simples (VOGT; KUHN, 2012b) e dos osciladores harmônicos amortecidos (CASTRO-PALACIO et al., 2013; KUHN; VOGT, 2012). CastroPalacio et al. (2013) utilizam o acelerômetro embutido do smartphone para coleta de dados em sistemas oscilantes com e sem amortecimento obtendo, assim, grandezas físicas como período, frequência, constante elástica da mola e constante de amortecimento do sistema. Já Kuhn e Vogt (2012), além das características principais do estudo de oscilações amortecidas introduzem o acoplamento de dois osciladores através do acelerômetro do smartphone.

Em análise aos trabalhos relacionados às oscilações amortecidas com uso de smartphone, percebe-se que em todos os casos as atividades experimentais propostas foram desenvolvidas com o uso de sofisticados equipamentos de laboratório como trilho de ar horizontal e contadores temporais, além do uso do próprio smartphone (CASTRO-PALACIO et al., 2013; KUHN; VOGT, 2012). Atividades deste tipo somente seriam possíveis em instituições de ensino que dispusessem de recursos financeiros suficientes para a compra de equipamentos de laboratório de empresas especializadas.

O presente trabalho traz uma proposta de atividade experimental com materiais de baixo custo e uso cotidiano, como uma mola, uma régua e um smartphone, para o estudo de oscilações amortecidas em regime subcrítico. O objetivo é mostrar que apesar da simplicidade dos materiais é possível obter resultados satisfatórios acerca de um experimento didático, mesmo na ausência de equipamentos especializados. A proposta é uma alternativa para professores de instituições que não possuem laboratório didático de física, além de aproximar o fazer científico da realidade dos alunos.

\section{FundamentaÇão TEÓRICA}

Um corpo pendurado em uma mola na direção vertical, quando posto a oscilar, acaba por parar, uma vez que a energia mecânica do sistema é dissipada por forças de atrito (ver Figura 1). Tal movimento é dito amortecido. Se o amortecimento é suficientemente grande o oscilador é incapaz de completar seu primeiro ciclo de oscilação, limitando-se a retornar ao equilíbrio com uma rapidez que se aproxima de zero à medida que o corpo se aproxima da posição de equilíbrio. Este tipo de movimento é dito superamortecido ou supercrítico. Se o amortecimento for suficientemente pequeno para que o sistema oscile com uma amplitude que diminui lentamente com o tempo o movimento é dito subamortecido ou subcrítico. O movimento com o mínimo amortecimento que ainda não resulta em oscilação é dito criticamente amortecido.

Nesse trabalho, nos limitamos a reproduzir e estudar, exclusivamente, o movimento de um sistema oscilante amortecido em regime subcrítico. Maiores informações sobre os regimes supercrítico e criticamente amortecido podem ser encontradas em Thornton e 
Marion (2011) e Zill e Cullen (2008).

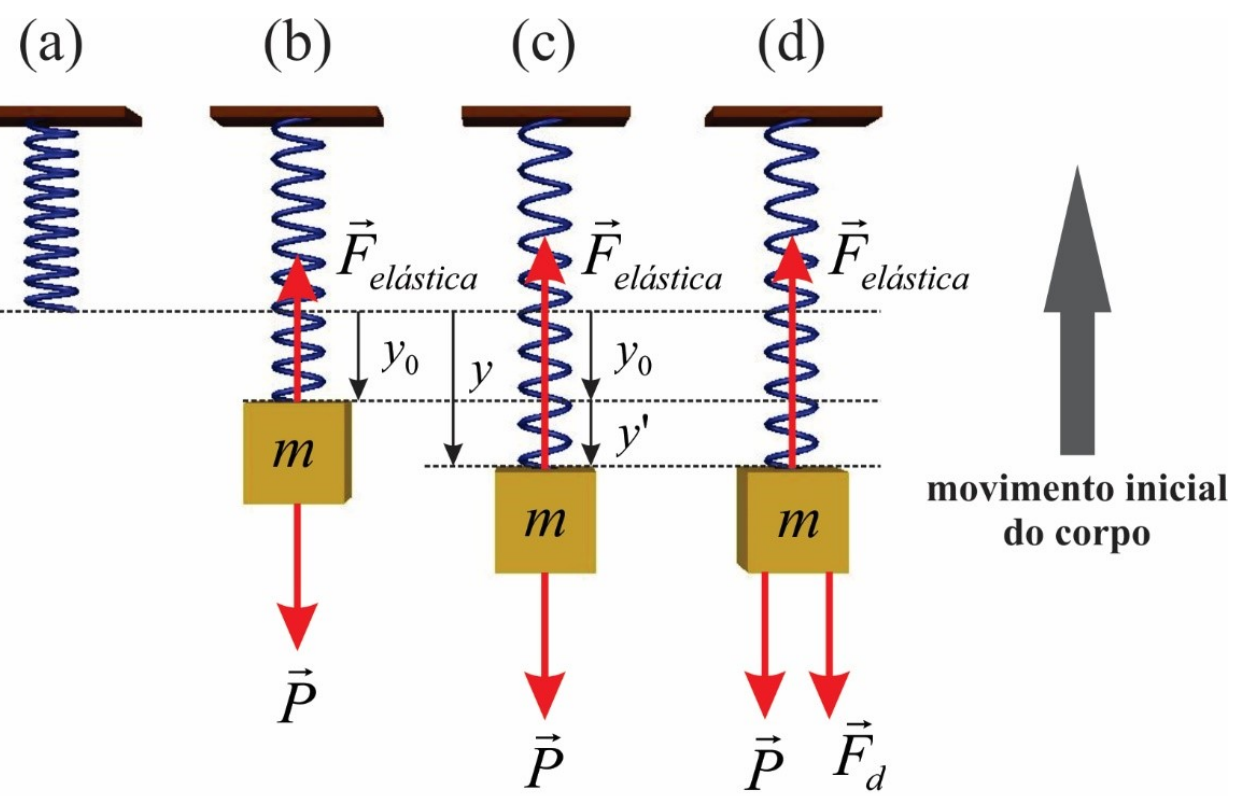

Figura 1: Em (a) temos a posição da extremidade inferior da mola relaxada. Em (b) o acoplamento do corpo de massa $m$ à mola produz o deslocamento do sistema para baixo até que uma nova posição de equilíbrio seja encontrada. Nesse momento, o peso do corpo $(\vec{P})$ e a força elástica $\left(\vec{F}_{\text {elástica }}\right)$ produzida pela mola se igualam e, portanto, a mola se distende de uma quantidade $y_{0}=m g / k$. Em (c) o corpo é deslocado, novamente, para baixo de $y^{\prime}$. Desse modo, a força elástica se torna maior que a força peso. Como consequência, no momento em que o corpo é solto, o movimento se dá na direção vertical, para cima. Em (d) uma força dissipativa $\vec{F}_{d}$ surge na direção contrária ao movimento do corpo. (Fonte: elaborado pelos autores)

A força de amortecimento $\vec{F}_{d}$ exercida sobre um oscilador como o mostrado na Figura 1 pode ser representada na expressão empírica

$$
\vec{F}_{d}=-\beta \vec{v}_{y}
$$

onde $\beta$ é uma constante de amortecimento positiva e o sinal negativo indica que a força de amortecimento atua em direção oposta à velocidade $\vec{v}_{y}$ do sistema. Se o sistema se desloca na direção vertical para cima, a força de amortecimento surge na direção vertical para baixo, como mostrado na Figura 1; se o sistema se move na direção vertical para baixo, a força de amortecimento surge na direção vertical para cima.

O movimento de um sistema amortecido pode ser obtido através da segunda lei de Newton (ver Apêndice I). As equações da posição ${ }^{1}$, velocidade e aceleração para um sistema oscilante em regime subcrítico são dadas por

$$
y^{\prime}(t)=y_{0}^{\prime} e^{-\lambda t}\left[\cos \left(\sqrt{\omega^{2}-\lambda^{2}} t\right)-\frac{\sqrt{\omega^{2}-\lambda^{2}}}{\lambda^{2}-\omega^{2}} \sin \left(\sqrt{\omega^{2}-\lambda^{2}} t\right)\right]
$$

\footnotetext{
${ }^{1} y^{\prime}$ não é a derivada primeira da posição. O apóstrofo é utilizado para indicar mudança de variável na demonstração da equação (2) (ver Apêndice I).
} 


$$
\begin{gathered}
v_{y}(t)=-\frac{y_{0}^{\prime} \omega^{2} e^{-\lambda t} \sin \left(\sqrt{\omega^{2}-\lambda^{2}} t\right)}{\sqrt{\omega^{2}-\lambda^{2}}} \\
a_{y}(t)=-\frac{y_{0}^{\prime} \omega^{2} e^{-\lambda t}\left[\cos \left(\sqrt{\omega^{2}-\lambda^{2}} t\right)-\cos \left(\sqrt{\omega^{2}-\lambda^{2}} t\right) \omega^{2}+\sqrt{\omega^{2}-\lambda^{2}} \lambda \sin \left(\sqrt{\omega^{2}-\lambda^{2}} t\right)\right]}{\lambda^{2}-\omega^{2}}
\end{gathered}
$$

onde $y_{0}^{\prime}$ é a amplitude inicial de oscilação, $\lambda$ é um parâmetro associado à constante de amortecimento $\beta$ e à massa $m$ do corpo e $\omega$ é a frequência natural de oscilação associada à constante elástica $k$ e à massa $m$

$$
\lambda=\frac{\beta}{2 m} \quad ; \quad \omega=\sqrt{\frac{k}{m}}
$$

Os gráficos das equações (2), (3) e (4) encontram-se na Figura 2.

(a)

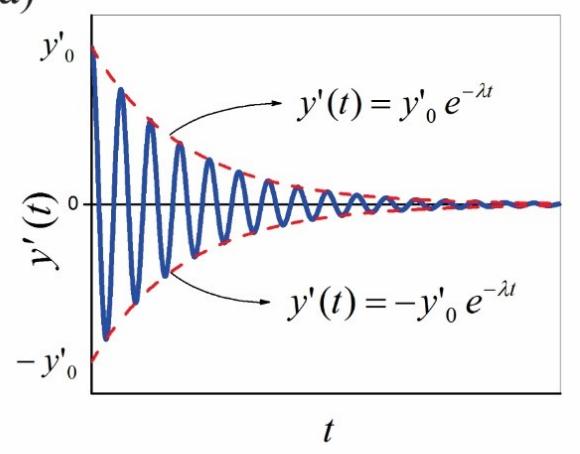

(b)

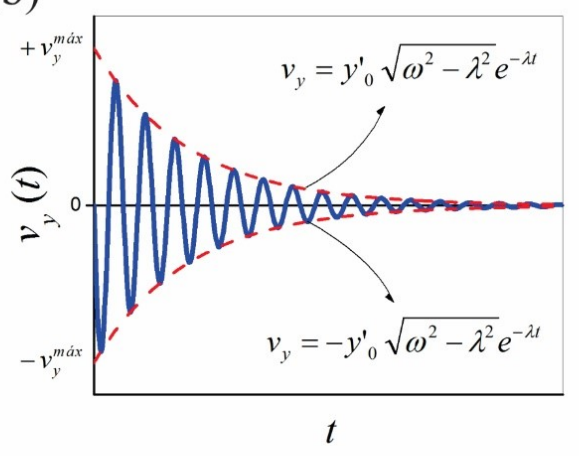

(c)

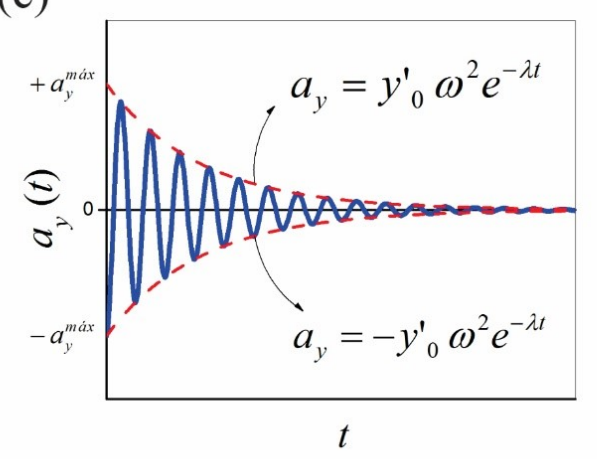

Figura 2: Gráficos do oscilador amortecido em regime subcrítico para (a) posição, (b) velocidade e (c) aceleração em função do tempo. (Fonte: elaborado pelos autores)

De acordo com a Figura 2a percebemos que o movimento de um oscilador amortecido em regime subcrítico difere do movimento de um oscilador harmônico simples no que se refere à manutenção do movimento. Um oscilador harmônico simples, uma vez em oscilação, jamais terá seu movimento interrompido. Esse fato é uma simplificação do caso real, no qual forças de dissipação irão finalmente amortecer o movimento até o ponto no 
qual nenhuma oscilação mais ocorrerá.

As envoltórias (curvas tracejadas em vermelho na Figura 2) que modelam o comportamento da posição, velocidade e aceleração do sistema no decorrer do tempo são representadas, respectivamente, pelas equações (6), (7) e (8).

$$
\begin{gathered}
y^{\prime}(t)= \pm y_{0}^{\prime} e^{-\lambda t} \\
v_{y}(t)= \pm y_{0}^{\prime} \sqrt{\omega^{2}-\lambda^{2}} e^{-\lambda t} \\
a_{y}(t)= \pm y_{0}^{\prime} \omega^{2} e^{-\lambda t}
\end{gathered}
$$

\section{MATERIAIS E MÉTODOS}

A proposta experimental deste trabalho consiste em analisar o movimento de um sistema oscilante na direção vertical formado por um smartphone da marca Samsung (modelo Galaxy S7, sistema operacional Android na versão 7.0) de massa $m=197,98 g$ acoplado à uma mola de constante elástica $k=7,053 \mathrm{~N} / m$ (ver Apêndice II para determinação da constante elástica da mola caso o seu valor nominal seja desconhecido). A montagem experimental foi realizada de acordo com a Figura 3.

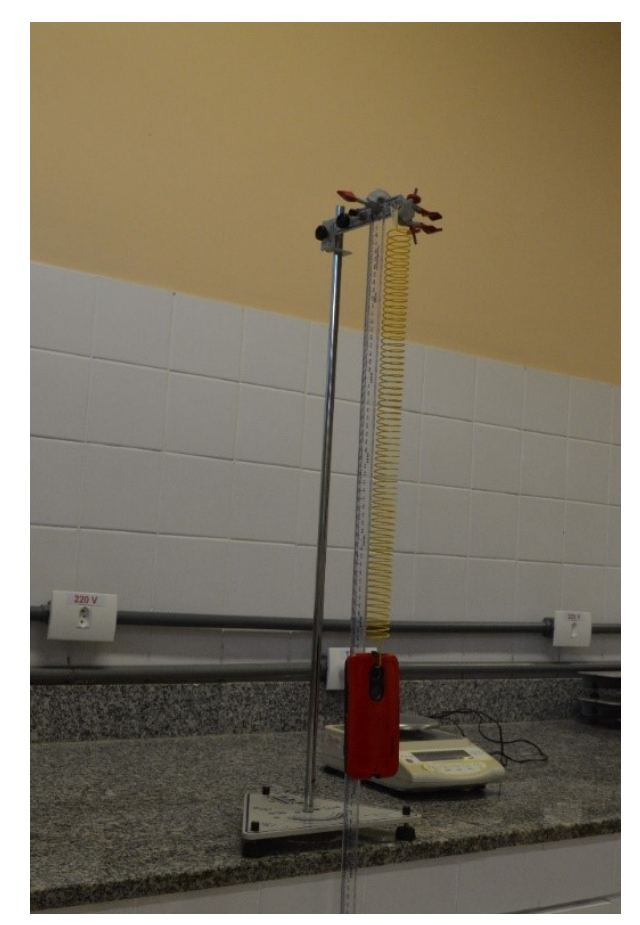

Figura 3: Montagem experimental. (Fonte: elaborado pelos autores)

A aquisição dos dados foi realizada de modo automático através do aplicativo Sensor Kinetics obtido através da Google Play do próprio aparelho smartphone (ver Figura 4a). Dentre as várias possibilidades de medida de diferentes grandezas físicas disponíveis no aplicativo, utilizamos o acelerômetro linear para medida da aceleração nos eixos $x, y$ e $z$ em função 
do tempo. Os eixos cartesianos $x, y$ e $z$ estão relacionados ao movimento do smartphone de acordo com a Figura $4 b$.

(a)

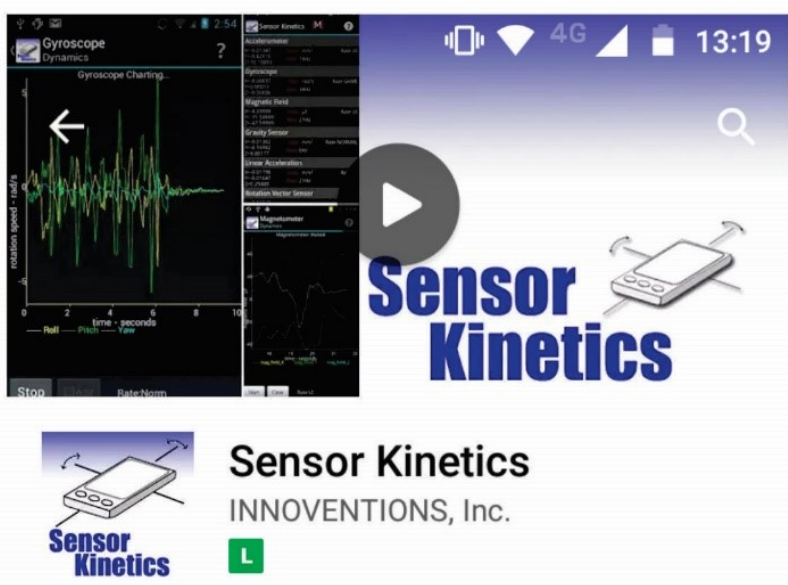

(b)

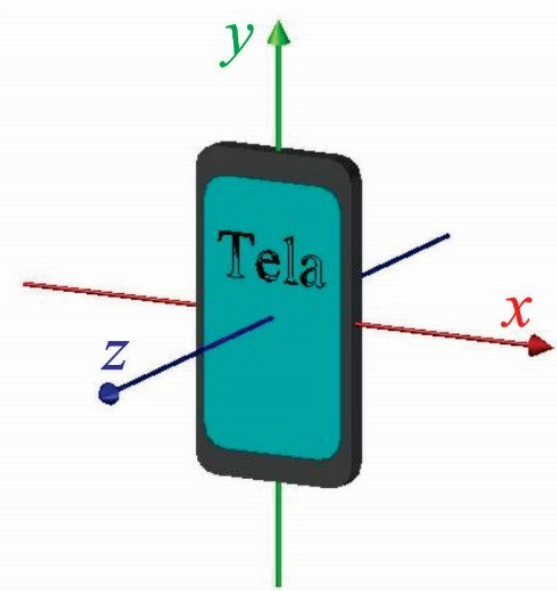

Figura 4: (a) aplicativo utilizado para registro da aceleração linear em função do tempo; (b) orientação dos eixos cartesianos $x$, y e $z$ relacionados ao movimento do smartphone. (Fonte: (a) Google Play e (b) elaborado pelos autores)

O sistema em equilíbrio (mola + celular) encontrava-se na posição $61,4 \mathrm{~cm}$. Para que o smartphone iniciasse seu movimento o deslocamos na direção vertical para baixo até a posição 51,4 cm e, então, o soltamos. Neste caso, a amplitude inicial do sistema foi igual a $51,4 \mathrm{~cm}-61,4 \mathrm{~cm}=-10,0 \mathrm{~cm}^{2}$. Iniciado o movimento do smartphone, deixamos o sistema oscilar por aproximadamente $450 \mathrm{~s}$.

Após a realização das medidas os dados foram salvos em uma tabela no formato CSV, no próprio aparelho smartphone, e exportados para o software Origin, versão 2018, para construção e análise dos gráficos.

\section{Resultados E discussão}

Os gráficos da aceleração relacionados aos eixos $x, y$ e $z$ podem ser vistos na Figura 5 .

Apesar de prepararmos o sistema para movimentar-se exclusivamente na direção vertical (eixo $y$ ), pequenas oscilações nos eixos $x$ e $z$ são observadas nos instantes iniciais (Figura 5a e Figura 5c). Tais oscilações são devidas ao modo como o smartphone é solto. Apesar disso, restringimos a análise do sistema oscilante somente ao eixo principal, no caso, o eixo y.

A curva e os parâmetros de ajuste podem ser vistos, respectivamente, na Figura 6 e na Tabela 1. O ajuste foi feito com base na equação (4) e com o auxílio do software Origin, versão 2018 (ORIGINLAB CORPORATION, 2018).

Tabela 1: Dados do ajuste da Figura 6.

\begin{tabular}{ccccc}
\hline Ajuste & $R^{2}$ & $y_{0}^{\prime}[\mathrm{m}]$ & $\lambda[\mathrm{N} \cdot \mathrm{s} / \mathrm{m} \cdot \mathrm{kg}]$ & $\omega[\mathrm{rad} / \mathrm{s}]$ \\
\hline Equação (4) & 0,96482 & 0,09395 & 0,0055 & 5,84586 \\
\hline
\end{tabular}

\footnotetext{
${ }^{2} \mathrm{O}$ sinal negativo está relacionado ao posicionamento da régua, que, neste caso, permaneceu com a escala de valores decrescente no sentido para baixo.
} 


\section{Revista do Professor de Física, v. 3, n. 2, p. 65-79, Brasília, 2019.}

(a)

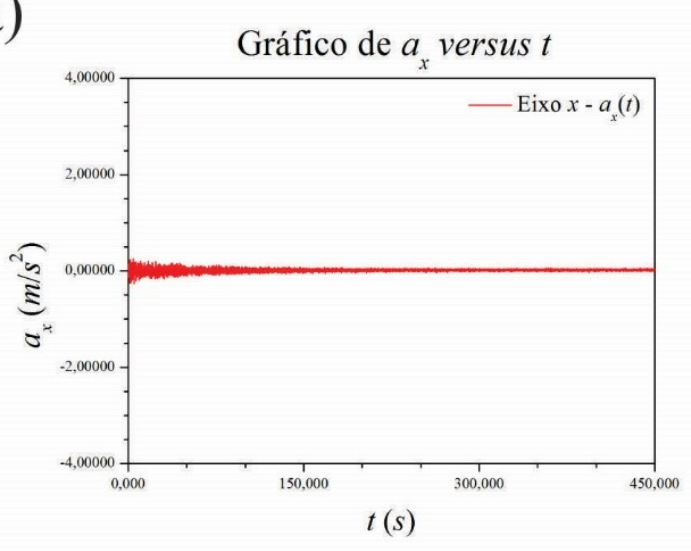

(b)

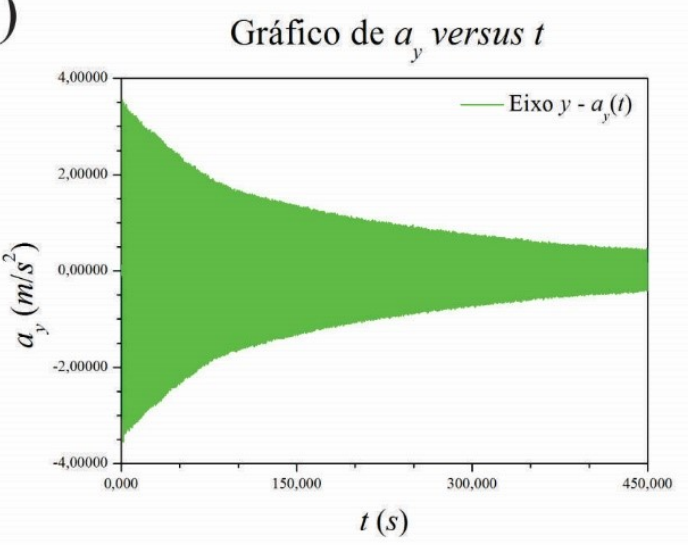

(c)

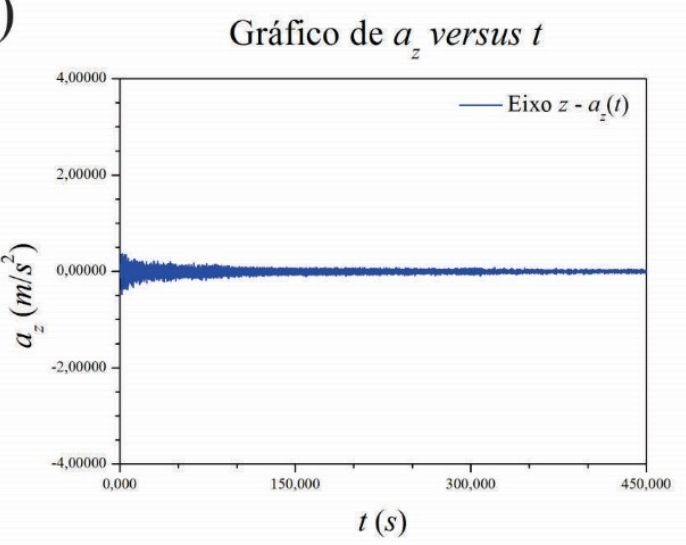

Figura 5: Medidas de aceleração versus tempo para os eixos (a) $x,(b)$ y e (c) z. (Fonte: elaborado pelos autores)

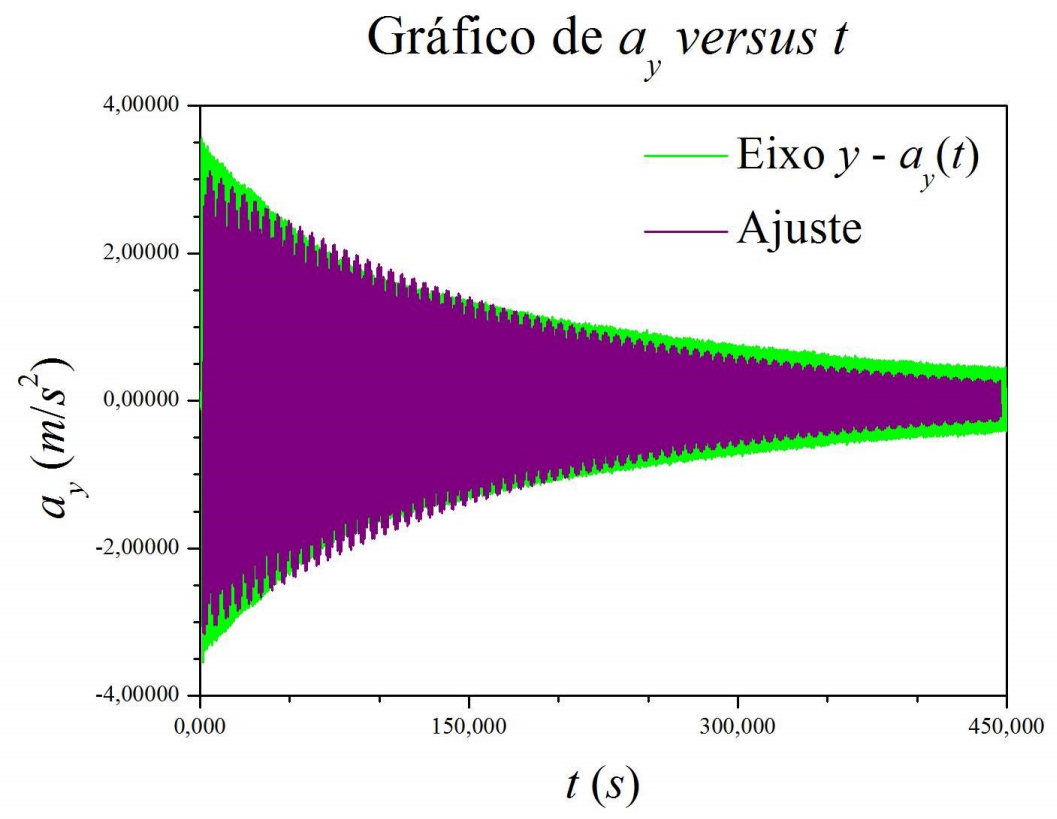

Figura 6: $a_{y}$ versus te ajuste dos pontos experimentais. (Fonte: elaborado pelos autores) 
Comparando o gráfico de $a_{y}(t)$ (Figura $5 \mathrm{~b}$ e Figura 6) obtido experimentalmente com o modelo teórico do oscilador amortecido (Figura 2c) observa-se semelhança entre as figuras, o que confirma o comportamento subcriticamente amortecido do sistema oscilante.

Substituindo os valores de $m$ e os valores de $\lambda$ e $\omega$ da Tabela 1 nas equações em (5) encontramos os valores do coeficiente de amortecimento $\beta=2 \lambda \mathrm{m}=2 \cdot(0,0055 \mathrm{~N} \cdot \mathrm{s} / \mathrm{m} \cdot \mathrm{kg})$. $(0,19798 \mathrm{~kg}) \approx 0,022 \mathrm{~N} \cdot \mathrm{s} / \mathrm{m}$ e da constante elástica da mola $k=m \omega^{2}=(0,19798 \mathrm{~kg})$. $(5,84586 \mathrm{rad} / \mathrm{s})^{2} \approx 6,7658 \mathrm{~N} / \mathrm{m}$.

O valor de $k \approx 6,7658 \mathrm{~N} / \mathrm{m}$, obtido através dos parâmetros de ajuste do gráfico da Figura 6 (com $\left.R^{2}=0,96482\right)$, difere em aproximadamente $4,07 \%$ do valor de $k \approx 7,053 \mathrm{~N} / \mathrm{m}$ obtido através da calibração da mola (com $R^{2}=0,99999$ - ver Apêndice II).

Ainda com os valores de $y_{0}^{\prime}, \lambda$ e $\omega$ da Tabela 1 e com as equações (6), (7) e (8) é possível construir, respectivamente, os gráficos das envoltórias superiores (positivas) e inferiores (negativas) da posição, velocidade e aceleração, que modelam o comportamento destas grandezas no decorrer do tempo (ver Figura 7). As envoltórias são curvas constituídas por pontos máximos e mínimos que cada grandeza física assume durante o movimento. $\mathrm{Na}$ Figura 7 percebe-se claramente a influência do fator $e^{-\lambda t}$, encontrado nas equações (6), (7) e (8), no decaimento exponencial da posição, velocidade e aceleração, o que evidencia a perda de energia quando forças dissipativas atuam em um sistema oscilante. O mesmo não acontece para o oscilador harmônico simples cuja envoltória são retas paralelas ao eixo temporal.

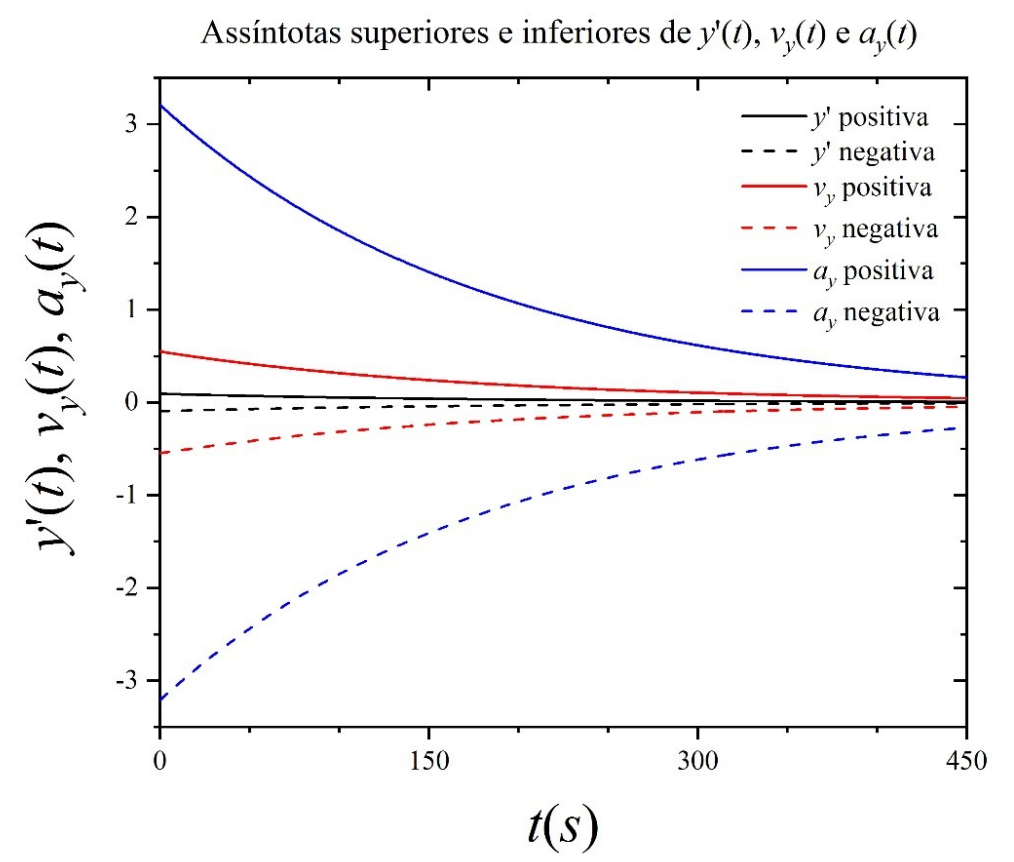

Figura 7: Envoltórias superiores e inferiores da posição, velocidade e aceleração. (Fonte: elaborado pelos autores) 


\section{CONSIDERAÇÕES FINAIS}

Os resultados encontrados na seção IV permitem as seguintes considerações:

A escolha de uma mola e um smartphone revelou-se suficiente para a realização de um experimento de boa qualidade, baixo custo e uso cotidiano para o estudo de oscilações amortecidas em regime subcrítico. Mesmo em instituições onde não exista laboratório de física, o professor pode reproduzir o experimento na própria sala de aula, sem a necessidade de sofisticados equipamentos ou sensores.

Em colaboração com o acelerômetro, o software Sensor Kinetics provou ser uma ferramenta eficiente para a obtenção simultânea de medidas de aceleração em função do tempo nos três eixos espaciais. Com esses resultados foi possível determinar os valores da constante de amortecimento $\beta$ e da constante elástica $k$ da mola, com acurácia e precisão aceitáveis para o propósito do trabalho.

A contribuição deste trabalho ao ensino de física consiste em apresentar um experimento simples e barato para o estudo de oscilações amortecidas em regime subcrítico e, através da análise dos dados, determinar os valores da constante de amortecimento e da constante elástica da mola previstos na teoria. Além disso, é possível observar o comportamento da envoltória da posição, velocidade e aceleração demonstrando, desta maneira, a perda de energia em um movimento oscilatório quando forças dissipativas atuam sobre o sistema.

Acreditamos que as atividades aqui desenvolvidas possam servir de incentivo à prática experimental de oscilações em nível superior, na forma apresentada nesse artigo, bem como no ensino médio, nesse caso empregando a abordagem aqui descrita de forma predominantemente qualitativa, dando assim aos estudantes a oportunidade de vivenciarem um pouco mais as dificuldades e minúcias inerentes ao conhecimento científico.

\section{REFERÊNCIAS}

CASTRO-PALACIO, J. C. et al. Using a mobile phone acceleration sensor in physics experiments on free and damped harmonic oscillations. American Journal of Physics, v. 81, n. 6 , p. $472-475$, jun 2013. 67

HAWLEY, S. H.; MCCLAIN, R. E. Visualizing sound directivity via smartphone sensors. The Physics Teacher, v. 56, n. 2, p. 72-74, feb 2018. 67

IGOE, D. P. et al. Median filters as a tool to determine dark noise thresholds in high resolution smartphone image sensors for scientific imaging. Review of Scientific Instruments, v. 89, n. 1, p. 015003 , jan 2018. 67

KUHN, J.; VOGT, P. Analyzing spring pendulum phenomena with a smart-phone acceleration sensor. The Physics Teacher, v. 50, n. 8, p. 504-505, nov 2012. 67

MENDES, A.; ROSÁRIO, P. P. Metrologia e incerteza de medição. 1. ed. Sao Paulo: EPSE, 2005. 128 p. 78

MONTEIRO, M. et al. Acceleration measurements using smartphone sensors: dealing with the equivalence principle. Revista Brasileira de Ensino de Física, v. 37, n. 1, p. 1303, mar 2015. 66 
MONTEIRO, M. et al. Magnetic field 'flyby' measurement using a smartphone's magnetometer and accelerometer simultaneously. The Physics Teacher, v. 55, n. 9, p. 580-581, dec 2017. 67

ORIGINLAB CORPORATION. Origin. Northampton: [s.n.], 2018. Disponível em: $<$ https://www.originlab.com/>. 71

PEREIRA, V. et al. Studying 3D collisions with smartphones. The Physics Teacher, v. 55, n. 5, p. 312-313, may 2017. 67

SALINAS, I. et al. Characterization of linear light sources with the smartphone's ambient light sensor. The Physics Teacher, v. 56, n. 8, p. 562-563, nov 2018. 67

SILVA, R. V.; SILVA, F. A. Utilização de dispositivos móveis com acelerômetro para controle de aplicações. Colloquium Exactarum, v. 2, n. 1, p. 12-20, 2010. 66

THORNTON, S. T.; MARION, J. B. Dinâmica clássica de partículas e sistemas. 1. ed. Sao Paulo: Cengage Learning, 2011. 608 p. 68

VOGT, P.; KUHN, J. Analyzing free fall with a smartphone acceleration sensor. The Physics Teacher, v. 50, n. 3, p. 182-183, mar 2012. 67

VOGT, P.; KUHN, J. Analyzing simple pendulum phenomena with a smartphone acceleration sensor. The Physics Teacher, v. 50, n. 7, p. 439-440, oct 2012. 67

ZILL, D. G.; CULLEN, M. R. Equações diferenciais - vol. 1. 3. ed. Sao Paulo: Pearson Makron Books, 2008. 473 p. $68,76,77$

\section{APÊNDICE}

I. Demonstração das equações da posição, velocidade e aceleração de um oscilador amortecido em regime subcrítico

Considere um corpo de massa $m$ acoplado verticalmente à mola de constante elástica $k$ da Figura 1.

Assumindo o sentido positivo de $y$ para baixo, a força resultante que atua sobre o corpo em movimento é, então

$$
\sum \vec{F}_{y}=\vec{F}_{\text {elástica }}+\vec{P}+\vec{F}_{d}=-k y+m g-\beta v_{y}
$$

onde $\sum \vec{F}_{y}$ é o somatório das forças que atuam sobre o corpo na direção $y, \vec{F}_{\text {elástica }}$ é a força exercida pela mola sobre o corpo, $\vec{P}$ o peso do corpo, $\vec{F}_{d}$ a força dissipativa que atua em oposição ao movimento do corpo, y é o deslocamento sofrido pela mola desde a configuração da Figura 1a até a configuração da Figura 1c, $g$ a aceleração da gravidade, $\beta$ a constante de amortecimento e $v_{y}$ a velocidade do corpo na direção $y$. 
Podemos simplificar a equação (9) mudando para uma nova variável $y^{\prime}=y-y_{0}$, onde $y_{0}=m g / k$ é o quanto a mola é distendida quando o corpo está em equilíbrio estático (Figura 1b). Substituindo $y$ por $y^{\prime}+y_{0}$, fica

$$
\sum F_{y}=-k\left(y^{\prime}+y_{0}\right)+m g-\beta v_{y}
$$

Mas $k y_{0}=m g$, de modo que

$$
\sum F_{y}=-k y^{\prime}-k y_{0}+m g-\beta v_{y}=-k y^{\prime}-m g+m g-\beta v_{y}=-k y^{\prime}-\beta v_{y}
$$

A segunda lei de Newton $\left(\sum F_{y}=m a_{y}\right)$ nos dá

$$
-k y^{\prime}-\beta v_{y}=m \frac{d^{2} y}{d t^{2}}
$$

No entanto, $y^{\prime}=y-y_{0}$, onde $y_{0}=m g / k$ é uma constante. Assim, $d^{2} y / d t^{2}=d^{2} y^{\prime} / d t^{2}$, de modo que

$$
-k y^{\prime}-\beta v_{y}=m \frac{d^{2} y^{\prime}}{d t^{2}}
$$

ou

$$
m \frac{d^{2} y^{\prime}}{d t^{2}}=-k y^{\prime}-\beta \frac{d y^{\prime}}{d t}
$$

Dividindo a equação (14) pela massa $m$, temos a equação diferencial do movimento amortecido (ZILL; CULLEN, 2008).

$$
\frac{d^{2} y^{\prime}}{d t^{2}}+\frac{\beta}{m} \frac{d y^{\prime}}{d t}+\frac{k}{m} y^{\prime}=0
$$

ou

$$
\frac{d^{2} y^{\prime}}{d t^{2}}+2 \lambda \frac{d y^{\prime}}{d t}+\omega^{2} y^{\prime}=0
$$

na qual realizamos as seguintes identificações na equação (16)

$$
2 \lambda=\frac{\beta}{m} \quad ; \quad \omega^{2}=\frac{k}{m}
$$

O símbolo $2 \lambda$ é usado somente por conveniência algébrica, pois a equação auxiliar é $r+2 \lambda r+\omega^{2}$ e as raízes correspondentes são, portanto,

$$
r_{1}=-\lambda+\sqrt{\lambda^{2}-\omega^{2}} \quad ; \quad r_{2}=-\lambda-\sqrt{\lambda^{2}-\omega^{2}}
$$

Podemos distinguir três casos possíveis, dependendo do sinal do termo $\lambda^{2}-\omega^{2}$ : se $\lambda^{2}-\omega^{2}>0$ o sistema é superamortecido, se $\lambda^{2}-\omega^{2}=0$ o sistema é criticamente amortecido e se $\lambda^{2}-\omega^{2}<0$ o sistema é subamortecido. Cada solução contém o fator de amortecimento $e^{-\lambda t}$. Sendo $\lambda$ um parâmetro positivo, o deslocamento da massa torna-se desprezível após um longo período de tempo. 
Neste trabalho nos restringimos ao caso subamortecido no qual $\lambda^{2}-\omega^{2}<0$. Sendo assim, temos que $\lambda^{2}<\omega^{2}$, ou seja,

$$
\frac{\beta^{2}}{4 m^{2}}<\frac{k}{m}
$$

A equação (19) nos mostra que o coeficiente de amortecimento $\beta$ é pequeno quando comparado à constante de amortecimento $k: \beta<2 \sqrt{m k}$. Neste caso, as raízes $r_{1}$ e $r_{2}$ da equação (18) serão complexas:

$$
r_{1}=-\lambda+\sqrt{\lambda^{2}-\omega^{2}} i \quad ; \quad r_{2}=-\lambda-\sqrt{\lambda^{2}-\omega^{2}} i
$$

Portanto a solução geral para a equação (16) é (ZILL; CULLEN, 2008):

$$
y^{\prime}(t)=e^{-\lambda t}\left[C_{1} \cos \left(\sqrt{\omega^{2}-\lambda^{2}} t\right)+C_{2} \sin \left(\sqrt{\omega^{2}-\lambda^{2}} t\right)\right]
$$

onde $C_{1}=y_{0}^{\prime}$ e $C_{2}=-\frac{y_{0}^{\prime} \sqrt{\omega^{2}-\lambda^{2}}}{\lambda^{2}-\omega^{2}}$ para as condições de contorno $y^{\prime}(0)=y_{0}^{\prime}$ e $v_{y}(0)=0$.

O movimento descrito pela equação (21) é oscilatório. Entretanto, devido ao fator exponencial $e^{-\lambda t}$, a amplitude de oscilação diminui com o passar do tempo. Em outras palavras $y_{0}^{\prime} \rightarrow 0$, quando $t \rightarrow \infty$.

A velocidade $v_{y}$ e a aceleração $a_{y}$ do sistema oscilante são dadas, respectivamente, pelas derivadas primeira $\left(d y_{0}^{\prime} / d t\right)$ e segunda $\left(d^{2} y_{0}^{\prime} / d t^{2}\right)$ de $y^{\prime}$ em relação a $t$ :

$$
\begin{gathered}
v_{y}(t)=-\frac{y_{0}^{\prime} \omega^{2} e^{-\lambda t} \sin \left(\sqrt{\omega^{2}-\lambda^{2}} t\right)}{\sqrt{\omega^{2}-\lambda^{2}}} \\
a_{y}(t)=-\frac{y_{0}^{\prime} \omega^{2} e^{-\lambda t}\left[\cos \left(\sqrt{\omega^{2}-\lambda^{2}} t\right)-\cos \left(\sqrt{\omega^{2}-\lambda^{2}} t\right) \omega^{2}+\sqrt{\omega^{2}-\lambda^{2}} \lambda \sin \left(\sqrt{\omega^{2}-\lambda^{2}} t\right)\right]}{\lambda^{2}-\omega^{2}}
\end{gathered}
$$

\section{Calibração da mola}

Para a determinação da constante elástica $k$ da mola realizamos a montagem experimental de modo semelhante ao da Figura 3 substituindo o smartphone por massas para calibração. Registramos a posição da extremidade inferior da mola para de diferentes valores de massa nela acoplada (ver Tabela 2). As medidas foram realizadas com o sistema em equilíbrio estático, pois, neste caso, tem-se $P=F_{\text {elástica }}$. A última coluna da Tabela 2 representa a diferença entre a posição da extremidade inferior da mola com massa acoplada e a posição de relaxamento (sem massa acoplada) ${ }^{3}$. A medida da posição da extremidade inferior da mola no estado de relaxamento $y_{0}$ foi de $(89,0 \pm 0,2) \mathrm{cm}=(0,890 \pm 0,002) \mathrm{m}$.

\footnotetext{
${ }^{3} \mathrm{O}$ sinal negativo está relacionado ao posicionamento da régua, que, neste caso, permaneceu com a escala de valores decrescentes no sentido para baixo.
} 
Tabela 2: Valores das massas para calibração e da posição da extremidade inferior da mola.

\begin{tabular}{cccccc}
\hline Massa & $m \pm \delta m[g]$ & $m \pm \delta m[\mathrm{~kg}]$ & $y \pm \delta y[\mathrm{~cm}]$ & $y \pm \delta y[m]$ & $\Delta y \pm \delta \Delta y[m]$ \\
\hline 1 & $29,99 \pm 0,001$ & $0,02999 \pm 0,00001$ & $84,8 \pm 0,2$ & $0,848 \pm 0,002$ & $-0,042 \pm 0,003$ \\
2 & $52,97 \pm 0,001$ & $0,05297 \pm 0,00001$ & $81,6 \pm 0,2$ & $0,816 \pm 0,002$ & $-0,074 \pm 0,003$ \\
3 & $75,98 \pm 0,001$ & $0,07598 \pm 0,00001$ & $78,4 \pm 0,2$ & $0,784 \pm 0,002$ & $-0,106 \pm 0,003$ \\
4 & $98,95 \pm 0,001$ & $0,09895 \pm 0,00001$ & $75,2 \pm 0,2$ & $0,752 \pm 0,002$ & $-0,138 \pm 0,003$ \\
5 & $121,94 \pm 0,001$ & $0,12194 \pm 0,00001$ & $72,0 \pm 0,2$ & $0,720 \pm 0,002$ & $-0,170 \pm 0,003$ \\
6 & $144,93 \pm 0,001$ & $0,14493 \pm 0,00001$ & $68,8 \pm 0,2$ & $0,688 \pm 0,002$ & $-0,202 \pm 0,003$ \\
7 & $167,92 \pm 0,001$ & $0,16792 \pm 0,00001$ & $65,6 \pm 0,2$ & $0,656 \pm 0,002$ & $-0,234 \pm 0,003$ \\
8 & $190,89 \pm 0,001$ & $0,19089 \pm 0,00001$ & $62,4 \pm 0,2$ & $0,624 \pm 0,002$ & $-0,266 \pm 0,003$ \\
9 & $213,87 \pm 0,001$ & $0,21387 \pm 0,00001$ & $59,3 \pm 0,2$ & $0,593 \pm 0,002$ & $-0,297 \pm 0,003$ \\
10 & $236,85 \pm 0,001$ & $0,23685 \pm 0,00001$ & $56,0 \pm 0,2$ & $0,560 \pm 0,002$ & $-0,330 \pm 0,003$ \\
\hline
\end{tabular}

Para o cálculo da incerteza de $\Delta y$ utilizamos a equação ${ }^{4}$

$$
\delta \Delta y=\sqrt{\left(\frac{\partial \Delta y}{\partial y} \delta y\right)^{2}+\left(\frac{\partial \Delta y}{\partial y_{0}} \delta y_{0}\right)^{2}}
$$

Sendo $\Delta y$ a diferença entre as posições final $y$ e inicial $y_{0}\left(\Delta y=y-y_{0}\right)$, as derivadas parciais $\partial \Delta y / \partial y$ e $\partial \Delta y / \partial y_{0}$ assumem, respectivamente, os valores 1 e -1 . Assim, a equação da incerteza acima pode ser escrita na forma

$$
\delta \Delta y=\sqrt{(\delta y)^{2}+\left(\delta y_{0}\right)^{2}}=\sqrt{(0,002)^{2}+(0,002)^{2}} \approx 0,003 m
$$

O gráfico de $m$ versus $\Delta y$ (em módulo) dos pontos experimentais da Tabela 2 pode ser visto na Figura 8. Considerando que todas as medidas foram realizadas com o sistema massa-mola em equilíbrio estático $\left(P=F_{\text {elástica }} \Rightarrow m g=k \Delta y\right)$, o valor da constante elástica $k$ da mola pode ser encontrado através do ajuste dos pontos experimentais por uma função de primeiro grau do tipo

$$
m=\frac{k}{g} \Delta y \Rightarrow m=a \cdot \Delta y
$$

onde a razão $k / g$ representa o coeficiente angular $a$ da equação, sendo $g$ a aceleração da gravidade local.

Os parâmetros obtidos através do ajuste encontram-se na Tabela 3.

Tabela 3: Dados do ajuste linear dos pontos experimentais do gráfico da Figura 8.

\begin{tabular}{cccccc}
\hline Eq. de ajuste & $R^{2}$ & $a[\mathrm{~kg} / \mathrm{m}]$ & $\delta a[\mathrm{~kg} / \mathrm{m}]$ & $b[\mathrm{~kg}]$ & $\delta b[\mathrm{~kg}]$ \\
\hline$y=a x+b$ & 0,99999 & 0,71922 & $3,44506 \cdot 10^{-5}$ & $-2,74332 \cdot 10^{-4}$ & $7,14255 \cdot 10^{-6}$ \\
\hline
\end{tabular}

Substituindo o valor da aceleração da gravidade local ao nível da bancada ${ }^{5} g=$

\footnotetext{
(2005).

${ }^{4} \mathrm{O}$ procedimento para o cálculo de propagação de incerteza pode ser encontrado em Mendes e Rosário

${ }^{5} \mathrm{O}$ valor da aceleração da gravidade ao nível da bancada foi obtido com auxílio do aplicativo Gravity Meter
} 


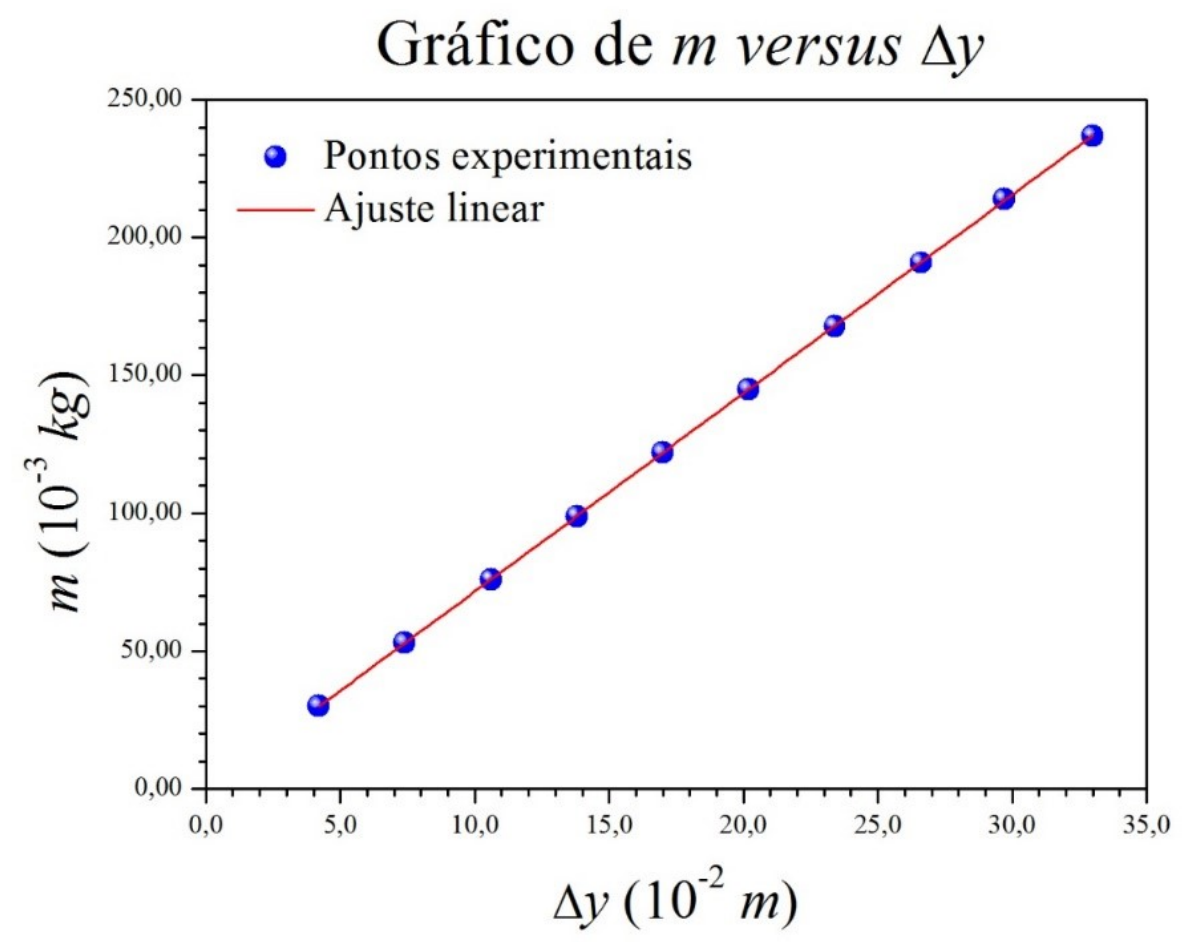

Figura 8: Gráfico dos pontos experimentais e ajuste linear dos dados da Tabela 2. (Fonte: elaborado pelos autores)

$(9,807 \pm 0,001) \mathrm{m} / \mathrm{s}^{2}$ e o valor do ajuste do coeficiente angular $a$ da Tabela 3 no termo $k=g \cdot a$, encontramos o valor da constante elástica $k$ da mola:

$$
k=7,053 \mathrm{~N} / \mathrm{m}
$$

A incerteza de $k$ foi calulada com base na equação

$$
\delta k=\sqrt{\left(\frac{\partial k}{\partial g} \delta g\right)^{2}+\left(\frac{\partial k}{\partial a} \delta a\right)^{2}}=\sqrt{(a \cdot \delta g)^{2}+(g \cdot \delta a)^{2}}
$$

Substituindo os valores de $a, \delta a, g$ e $\delta g$ na equação (28) encontramos

$$
\delta k=\sqrt{(0,71922 \cdot 0,001)^{2}+\left(9,807 \cdot 3,44506 \times 10^{-5}\right)^{2}} \approx 0,0000002 \mathrm{~N} / \mathrm{m}
$$

Apesar de calcularmos a incerteza da constante elástica, o seu valor foi desprezado, pois é muito pequeno quando comparado à quantidade de algarismos significativos de $k$. 